\title{
Molecular-Gated Drug Delivery Systems Using Light-Triggered Hydrophobic-to-Hydrophilic Switches
}

\author{
Ghani, Mozhdeh; Heiskanen, Arto; Thomsen, Peter; Alm, Martin; Emneus, Jenny
}

\section{Published in:}

ACS Applied Bio Materials

Link to article, DOI:

$10.1021 /$ acsabm.0c01458

Publication date:

2021

Document Version

Peer reviewed version

Link back to DTU Orbit

Citation (APA):

Ghani, M., Heiskanen, A., Thomsen, P., Alm, M., \& Emneus, J. (2021). Molecular-Gated Drug Delivery Systems Using Light-Triggered Hydrophobic-to-Hydrophilic Switches. ACS Applied Bio Materials, 4(2), 1624-1631. https://doi.org/10.1021/acsabm.0c01458

\section{General rights}

Copyright and moral rights for the publications made accessible in the public portal are retained by the authors and/or other copyright owners and it is a condition of accessing publications that users recognise and abide by the legal requirements associated with these rights.

- Users may download and print one copy of any publication from the public portal for the purpose of private study or research.

- You may not further distribute the material or use it for any profit-making activity or commercial gain

- You may freely distribute the URL identifying the publication in the public portal 


\title{
Molecular Gated Drug Delivery System Using Light-Triggered Hy- drophobic-to-Hydrophilic Switch
}

\author{
Mozhdeh Ghani ${ }^{1,2}$, Arto Heiskanen ${ }^{2}$, Peter Thomsen ${ }^{1}$, Martin Alm ${ }^{1 *}$, Jenny Emnéus²* \\ ${ }^{1}$ Biomodics ApS, Fjeldhammervej 15, 2610 Rødovre, Denmark \\ ${ }^{2}$ DTU Bioengineering, Building 423, 2800 Kgs. Lyngby, Denmark
}

\begin{abstract}
A photo-responsive molecule-gated drug delivery system (DDS) based on silicone-hydrogel (poly(HEMA-coPEGMEA)) interpenetrating polymer networks (IPN) functionalized with carboxylated spiropyran (SPCOOH) was designed and demonstrated as an on-demand DDS. The triggered release mechanism relies on controlling the wetting behaviour of the surface by light, exploiting different hydrophobicity between the "closed" and "open" isomers of spiropyran as the photo-switchable molecular gate on the surface of IPN (SP-photogated IPN). The light-triggered release of doxycycline (DOX) as the model drug indicated that the spiropyran (SP) molecules provide a hydrophobic layer around the drug carrier and have a good gate-closing efficiency for IPNs with 20-30 \% hydrogel content. Upon UV light irradiation the SP convert to the open hydrophilic merocyanine state, which triggers the release of DOX. These results were compared with a previously developed SP-bulk modified IPN using the same hydrogel as a control, proving the efficiency of the gated IPN system. The covalent attachment of SPCOOH to the alcohol groups of the hydrogel and the structural change caused by UV light was indicated with FTIR analysis. XPS results also confirms the presence of SP by indicating the atomic percentage of nitrogen with respect to the hydrogel content.
\end{abstract}

Keywords: Spiropyran, IPN, Molecular switch, Light-triggered release, Hydrophobicity switch.

\section{INTRODUCTION}

A major problem with conventional methods of drug administration is the lack of control over the drug concentration at the target site, the rate of drug release, and systematic side effects due to nonspecific biodistribution of the drug ${ }^{1}$. Ondemand DDS have the potential to address these issues by delivering the drug to a desired site of action, at a predetermined rate, and for a definite time duration, all of which enhances the therapeutic efficacy of the drug while reducing its toxicity ${ }^{1-6}$. Particularly, light-responsive DDS are considered advantageous in terms of having the potential for remote precise spatial and temporal control ${ }^{7}$ and ease of use ${ }^{8-11}$. Light can be easily switched 'off' and 'on', and the rate of drug release can be tuned remotely according to the duration of exposure ${ }^{12-14}$.

Among the available classes of light responsive molecular switches that can be used for developing the light-triggered DDS, SP ${ }^{15,16}$ has attracted a lot of attention ${ }^{17,18}$. The uniqueness of SP is due to the fact that the two isomers, spiropyran (SP) and merocyanine (MC), have extremely different physicochemical properties, moreover the response to light as the external stimuli is rapid and reversible ${ }^{17,18}$. At dark or under visible light the molecule is in its SP form, which is hydrophobic, colourless, uncharged, nonpolar, and insoluble in water. Under UV light irradiation, it isomerizes to the merocyanine (MC) form, which is hydrophilic, coloured (violet), zwitterionic, polar, and soluble in water ${ }^{19,20}$. Many efforts have aimed at developing technologies for reliable light-triggered release based on SP. This has been achieved by two strategies. One is introducing SP into amphiphilic block copolymers in which SP represents the hydrophobic segment ${ }^{21-29}$. These amphiphilic structures selfassemble into micelles in which the drug is encapsulated. UV light irradiation induces the isomerization of hydrophobic SP units to hydrophilic zwitterionic MC units, leading to the shift of the hydrophilic-hydrophobic balance, which consequently disassembles and disrupts the micellar structure and the encapsulated drug is released ${ }^{23,24,30}$. The second strategy uses SP as a gate molecule on the surface of drugloaded mesoporous silica ${ }^{31-34}$. Exploiting the hydrophobicity-hydrophilicity switch between the closed (SP) and open (MC) isomers allows control and adjustment of the wetting behaviour of the surface and release of drug.

In spite of the advancement in triggered release using these strategies, they are plagued by certain limitations and shortcomings that need to be addressed: First, most of these systems are designed just for hydrophobic drugs because they are mainly based on micelles with a hydrophobic core or a silica particle as the drug carrier. Second, the majority are based on micelles where the triggered-release mechanism is relying on disassembly and disruption of micelles, which means that the polymer components together with the drug will be released in the environment which is not always desirable due to the risk of toxic biproducts and haphazard side-reactions.

We have previously demonstrated the development of IPNbased drug delivery system by impregnation of silicone elastomers (as the host polymer) with different acrylicbased guest polymers using the supercritical carbon dioxide 
$\left(\mathrm{scCO}_{2}\right)$ technology ${ }^{35-41}$. Employing this technology, different types of monomers could be incorporated into the silicone to develop IPNs with various properties. We have shown that IPNs with the guest polymers of poly(2-hydroxyethyl methacrylate) (HEMA) and poly(2-hydroxyethyl methacrylate)-co-poly(ethylene glycol) methyl ether acrylate (poly(HEMA-co- PEGMEA)) has good potential for long-term continuous drug delivery ${ }^{39-41}$. Moreover, in a recent study ${ }^{42}$, we demonstrated that incorporation of different SP modified guest polymers (with different hydrophilicity) into the silicone elastomer, resulted in reversible photoresponsive SP-IPNs that could be used for delivery of different drugs by switching UV light on and off repetitively.

A SP-photogated IPN was fabricated by first impregnating poly(HEMA-co-PEGMEA) hydrogel into the silicone elastomer, followed by post-modification of the silicone-hydrogel IPN with SP. Light-triggered release and premature release of DOX was thereafter investigated by exposing the IPN to UV light. By grafting the surface of the IPN with hydrophobic gate molecules of carboxyl-containing SP (SPCOOH), the SP moieties act as the OFF state of the gate, preventing the drug from being released. Upon UV light irradiation at $375 \mathrm{~nm}$, the hydrophobic to hydrophilic switch converts hydrophobic SP to hydrophilic MC, which activates the $\mathrm{ON}$ state of the molecular gate. This leads to diffusion of water into the hydrogel with the capped drug, which swells and subsequent release of the drug.

\section{EXPERIMENTAL SECTION}

\subsection{Materials and chemicals}

Silicone hollow cylinders (L:22 mm, ID: $4 \mathrm{~mm}$, WT: $0.5 \mathrm{~g}$ ) were cut from extruded silicone tubing (Nusil MED-4020). Carbon dioxide (N48) was purchased from Air Liquide Denmark. HEMA $(97 \%)$, 4-(dimethylamino)pyridine (DMAP), PEGMEA (Mn 480), dicyclohexylcarbodiimide (DCC), 2,3,3-trimethylindolenin 98\%, tert-butyl methyl ether (TBME), 2-hydroxy-5-nitrobenzaldehyde, tetrahydrofuran (THF), 98\% ethylene glycol dimethacrylate (EGDMA), 3-iodo-propanoic acid, ethyl methyl ketone, piperidine, doxycycline hyclate (DOX) and ethanol (99.8\%, EtOH) were purchased from Sigma-Aldrich. The monomethyl ether hydroquinone (MEHQ), the polymerisation inhibitor of HEMA and PEGMEA, was removed as described previously [48]. Diethyl peroxydicarbonate (DEPDC) in hexane $(0.2 \mathrm{M})$ was prepared as described by $\mathrm{Xu}$ et al [56] and used as initiator.

\subsection{Fabrication of silicone-hydrogel IPNs}

The silicone-hydrogel IPNs were produced by impregnating silicone elastomer with hydrogel using $\mathrm{scCO}_{2}{ }^{35-41}$. IPNs containing $~ 50 \%, 40 \%, 30 \%$ and $20 \%$ (w/w) hydrogel were prepared in a $16 \mathrm{~mL}$-custom-made high-pressure reactor (Abeto, Copenhagen, Denmark). The reactor was loaded with a mixture of HEMA (1.6 mL), PEGMEA (1.6 mL), EGDMA (0.96 mL), ethanol (1.12 mL), THF (1.12 mL), DEPDC $(0.8 \mathrm{~mL})$ and approximately $0.5 \mathrm{~g}$ hollow cylinder silicone elastomer. Then, the reactor was closed and $\mathrm{CO}_{2}$ was added to a pressure of 360 bar at $45^{\circ} \mathrm{C}$; the reaction was continued for $16 \mathrm{~h}$ for complete impregnation and polymerization of poly(HEMA-co-PEGMEA) hydrogel into the silicone. The reactor was then cooled to ambient temperature and the pressure was slowly released; the samples were purified by extracting the residual monomers through soaking in $96 \%$ ethanol for 7 days. The hydrogel content of the IPNs was calculated using Eq. (1)

$$
G(\%)=\left(\frac{m_{I P N}-m_{\text {Silicone }}}{m_{I P N}}\right) \times 100
$$

Where, $m_{I P N}$ is the mass of the silicone-hydrogel IPN and $m_{\text {silicone }}$ is the mass of pristine silicone before impregnation with the hydrogel.

\subsection{Synthesis of carboxyl-containing spiropyran (SPCOOH)}

1-(b-carboxyethyl)-30,30-dimethyl-6-nitrospiro (indoline20,2[2H-1] benzopyran) (SPCOOH) was synthesized according to a slight modification of a previously described method $^{43}$. The reaction was performed at dark by wrapping the reaction vessels with aluminium foil during the synthesis. 3-iodo-propanoic acid (0.02 mol) and 2,3,3-trimethylindolenine $(0.02 \mathrm{~mol})$ were mixed with $15 \mathrm{~mL}$ of ethyl methyl ketone solution and refluxed for $3 \mathrm{~h}$ under nitrogen. After cooling to room temperature, the precipitated solid was collected and dissolved in water followed by washing with chloroform. The resulting iodide salt, 1-(b-carboxyethyl)2,3,3-trimethylindolenine iodide (4.5 g, $12.6 \mathrm{mmol})$, together with piperidine $(1.3 \mathrm{~mL}, 13.2 \mathrm{mmol})$ and 2-hydroxy5-nitrobenzaldehyde $(2.1 \mathrm{~g}, 12.6 \mathrm{mmol})$ were dissolved in $20 \mathrm{~mL}$ ethyl methyl ketone. The reaction mixture was refluxed for $3 \mathrm{~h}$ and then left overnight without stirring at room temperature. After cooling to $0^{\circ} \mathrm{C}$, a yellow crystalline solid was precipitated and washed with cold ethyl methyl ketone followed by methanol to obtain the product $\mathrm{SPCOOH}(72 \%$ yield $)$.

\subsection{Grafting of SPCOOH to IPN surface}

Surface modification of IPNs was performed by grafting of SPCOOH on the IPN surface using the Steglich esterification process similar to the method described in ${ }^{44}$. The HEMA alcohol groups on the IPN surface are the functional site for esterification with SPCOOH (Figure 1). To perform the surface grafting, DMAP, DCC and TBME were used as the esterification catalyst, dehydrating agent and solvent, respectively. A dry round-bottom flask was wrapped with aluminium foil. DCC (55 mg, $0.27 \mathrm{mmol})$, SPCOOH (100 $\mathrm{mg}, 0.27 \mathrm{mmol}$ ), DMAP (33 $\mathrm{mg}, 0.27 \mathrm{mmol}$ ) and $12 \mathrm{~mL}$ TBME were added to the flask. The flask was dried and flooded with nitrogen. Poly(HEMA-co-PEGMEA) IPNs were added to the mixture and gently stirred for 12 hours at room temperature. After the modification, the IPNs were washed with TBME, ethanol and water in an ultrasonic bath for 5 minutes each. Finally, the IPNs were dried at vacuum over molecular sieves for at least 5 hours.

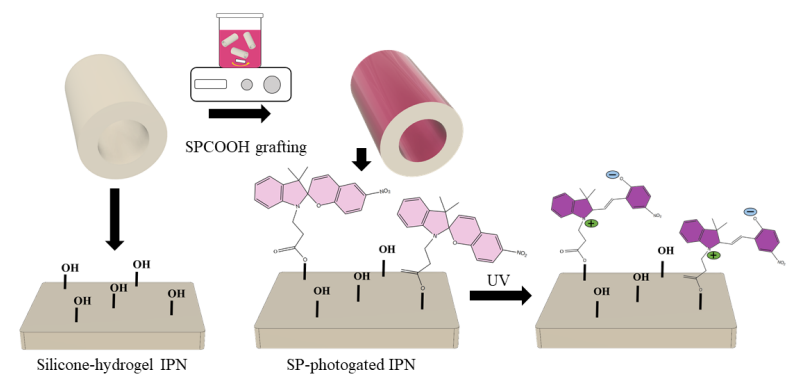


Figure 1. The schematic illustration of fabrication of SPphotogated IPN and isomerisation of hydrophobic SP to the hydrophilic MC upon UV irradiation.

\subsection{Characterization of SP-photogated IPNs}

The atomic composition of the surface and bulk of the SP grafted IPNs with different hydrogel content was analysed by X-ray photoelectron spectroscopy (XPS) using a K-Alpha spectrometer (Thermo Fisher Scientific Inc., USA) equipped with a monochromatic $\mathrm{Al} \mathrm{K \alpha} \mathrm{X}$-ray source (pass energy: $200 \mathrm{eV}$ for survey spectra). The atomic percentages of elements were extracted using the software package (Avantage Thermo VG) provided by Thermo Fisher Scientific.

Attenuated total reflection Fourier transform infrared spectroscopy (ATR-FTIR, PerkinElmer Technologies, USA) was used to identify different functional groups and verify the presence of hydrogel and SPCOOH grafted molecules. Moreover, FTIR was used to investigate the structural change caused by UV light.

To assess the change in wettability of the SPCOOH upon UV light irradiation, a $0.1 \mathrm{M}$ solution of SPCOOH in THF was prepared and coated on cover slides, which was followed by contact angle measurements before and after exposure to UV light. The measurement was performed using contact angle meter (Dataphysics, Germany). The measurement has performed on five different locations on each sample.

The water uptake of the SP-photogated IPN before and after irradiation with UV light was evaluated. Samples were kept in a desiccator for one day, then they were weighed $\left(w_{0}\right)$ and put into individual vials containing PBS ( $\mathrm{pH} 7.4)$. The samples were taken out at given time points, wiped gently to remove the excess water from the surface and weighed $\left(w_{t}\right)$. The samples were then transferred back to their vials. To study the swelling under UV light, samples were kept under a UV lamp (6 watt, $375 \mathrm{~nm}$, Analytik Jena, Germany). The water content of the samples was calculated using eq. (2).

$$
\text { water content }(\%)=\frac{w_{t}-w_{0}}{w_{t}} \times 100 \%
$$

2.6. Drug loading and drug release studies

The hollow cylinder SP-photogated IPNs $(1.00 \mathrm{~cm})$ were loaded by soaking in $5 \mathrm{~mL}$ DOX solution $(12.5 \mathrm{mg} / \mathrm{mL}$ in $70 \%$ ethanol) for 1 week at room temperature. Then, the samples were vortex-washed five times (10 sec each time) with fresh milli-Q water to remove loosely attached drug from the surface of samples. To investigate the triggered release under UV irradiation, the SP-photogated IPNs were immersed in vials containing $5 \mathrm{~mL}$ PBS $(0.1 \mathrm{M}, \mathrm{pH} 7.2)$. UV irradiation of the samples was done using a UV lamp (6-watt, $375 \mathrm{~nm}$, Analytik Jena, Germany). $300 \mu \mathrm{L}$ of the release medium was sampled from each vial at different time intervals. UV-Vis absorbance of the solution was then immediately recorded at the maximum absorption wavelength of DOX (344 nm).

\section{RESULTS AND DISCUSSION}

It is crucial for any light triggered DDS to inhibit the background release (premature release when light is off) and only deliver the drug on-demand at a certain point in time. In our previous work 42 a light-triggered DDS was designed based on incorporation of SP moieties inside the bulk of the IPN (SP-bulk IPN), where, the silicone elastomer was impregnated with methacrylated SP monomer (SPMA) copolymerized with different hydrophobic and hydrophilic acrylic monomers to develop photo-responsive IPNs with different hydrophilicity/hydrophobicity. It was demonstrated that the SP-bulk IPN with the most hydrophilic guest polymer (poly(HEMA-co-PEGMEA-SPMA)) showed the highest accumulated release, however with a similarly high premature release of DOX, indicating that the light triggered release was dysfunctional, which essentially was due to the swelling of guest polymer in absence of UV light. The more hydrophobic or partially hydrophilic SP-bulk IPNs (poly (BMA-co-SPMA) and poly(BMA-co-HEMA-SPMA guest polymers, respectively) demonstrated on the other hand nice light triggered DOX release with significantly lower premature release. The accumulated DOX release was however much lower than for the most hydrophilic SP-bulk IPN, most likely due to inefficient loading of the drug into the hydrophobic guest polymer. We therefore decided to instead explore the possibility to modify the surface of the hydrophilic poly(HEMA-co-PEGMEA) IPN by grafting SP on its surface as a molecular gate (SP-photogated IPN). It was hypothesized that this structure should have a high DOX loading efficiency but that the premature release could be inhibited by the hydrophobic SP molecular gate layer. Drug release should then be possible by opening the gate by light triggered isomerization of hydrophobic SP to hydrophilic MC.

\subsection{Characterization of SP-photogated IPNs}

Physical characteristics upon UV light exposure: Figure 2 shows the SP-photogated poly(HEMA-co-PEGMEA) IPNs with different hydrogel content before and after UV exposure. As seen, the colour of the samples changes upon UV exposure as a consequence of SP to $\mathrm{MC}$ isomerization. The more hydrogel content, the more purple the sample are, which is due to the increasing number of SP groups grafted on the surface. The coloration confirms that SP photo-isomerization was retained after the reaction, thereby confirming that the grafting does not adversely affect its reversible switching behavior.

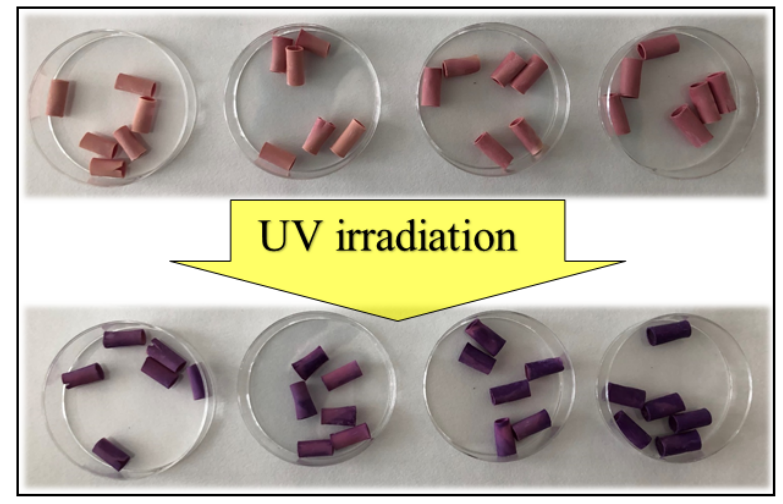

Figure 2. Surface grafted poly(HEMA-co-PEGMEA) IPNs before (top) and after (bottom) UV light irradiation (375 nm). The samples contain hydrogel content 20,30, 40 and $50 \mathrm{wt} \%$ from left to right, respectively.

FTIR spectra of IPNs before and after grafting: To identify the functional groups and confirm the presence of hydrogel in the silicone hydrogel IPNs and SP on the SP-grafted IPN surfaces, ATR-FTIR was used to characterize the samples. 
Figure 3 shows the ATR-FTIR spectra of pristine silicone and poly(HEMA-co-PEGMEA) IPNs with hydrogel contents of $20 \%$ and $50 \%(\mathrm{w} / \mathrm{w})$ before and after grafting with $\mathrm{SPCOOH}$. In the IPN spectra, the characteristic absorption peaks assigned to silicone are observed at $1008 \mathrm{~cm}^{-1}, 1258$ $\mathrm{cm}^{-1}$ and $2963 \mathrm{~cm}^{-1}$ and due to stretching vibrations of $\mathrm{Si}-$ $\mathrm{O}-\mathrm{Si}$, bending vibrations of $\mathrm{Si}-\mathrm{CH}_{3}$ and stretching vibrations of $\mathrm{CH}_{3}$ - groups, respectively. Moreover, the characteristic peaks around $1720 \mathrm{~cm}^{-1}$ and $3300 \mathrm{~cm}^{-1}$, which correspond to the ester carbonyl in the acrylate and the terminal $\mathrm{O}-\mathrm{H}$ groups of HEMA, respectively, confirm the presence of the HEMA in the IPNs. As expected, these peaks are broader and sharper in the IPN with 50\% hydrogel content. FTIR spectrum of IPNs after grafting with SP exhibits a band at $1341 \mathrm{~cm}^{-1}$ assigned to the $\mathrm{C}-\mathrm{O}$ ester group which confirms the SP esterification and the HEMA alcohol groups on the IPN surface.

FTIR spectra of SP grafted IPNs before and after UV light irradiation: FTIR was also used to investigate the structural change of the SP-photogated IPN due to UV light. The surface grafted poly(HEMA-co-PEGMEA) IPN with $20 \%$ (w/w) hydrogel content was used for this test, and UV irradiation was conducted with a 6-watt UV lamp (375 nm, Analytikjena) for 1 minute.

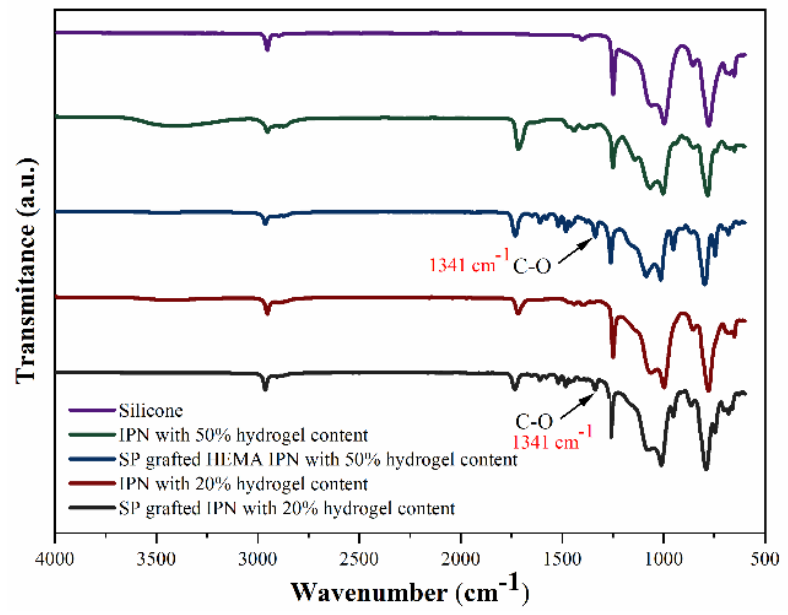

Figure 3. Transmission FTIR spectra of pristine silicone and poly(HEMA-co-PEGMEA) IPNs with different hydrogel content before and after grafting with SP.

The differential ATR-FTIR spectra was obtained by subtracting the spectra acquired before UV from that after UV exposure. It is believed that the ring-opening process of SP takes place after the irradiation, which results in breaking of the $\mathrm{C}-\mathrm{O}$ bonds ${ }^{18}$. As seen in Figure 4, the peaks at $947 \mathrm{~cm}^{-}$ ${ }^{1}$ and $1005 \mathrm{~cm}^{-1}$, corresponding to $\mathrm{C}_{\text {spiro }}-\mathrm{O}$ stretching ${ }^{45}$, appear in the ATR-FTIR differential spectra. The appearance of these peaks in the differential spectra is due to the decreased intensity of peaks corresponding to $\mathrm{C}_{\text {spiro }}-\mathrm{O}$ in the spectra of the SP-photogated IPN after exposure to UV light due to the cleavage of this bond.

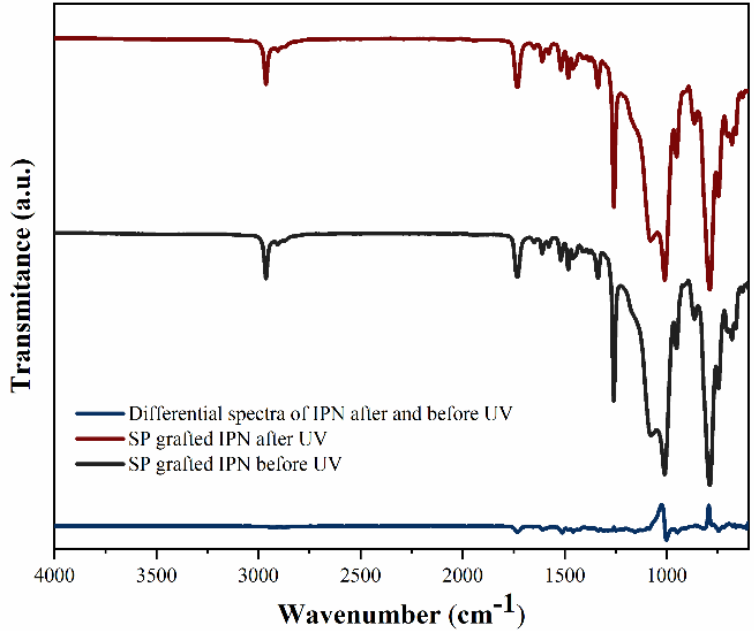

Figure 4. FTIR spectra of poly(HEMA-co-PEGMEA) IPN before and after UV exposure, and the corresponded differential spectra.

Elemental analysis of SP grafted IPNs: XPS analysis was used to verify that SP was grafted on the IPN surfaces with different hydrogel content. XPS can distinguish the nitrogen (N) atoms of SP and was therefore used to study their presence on the IPN surfaces and to evaluate the ratio of the grafted SP with respect to the hydrogel content. The XPS spectra of SP grafted IPNs with hydrogel contents of 20,30, 40 and $50 \%(\mathrm{w} / \mathrm{w})$ are shown in Figure 5. As seen, all the $\mathrm{SP}$ grafted IPNs show a nitrogen $\left(\mathrm{N}_{\mathrm{s}}\right)$ peak at $398 \mathrm{eV}$ with the atomic percentage increasing with increasing hydrogel content. This can be explained by the increasing number of HEME alcohol groups on the surface, available for esterification with SPCOOH.

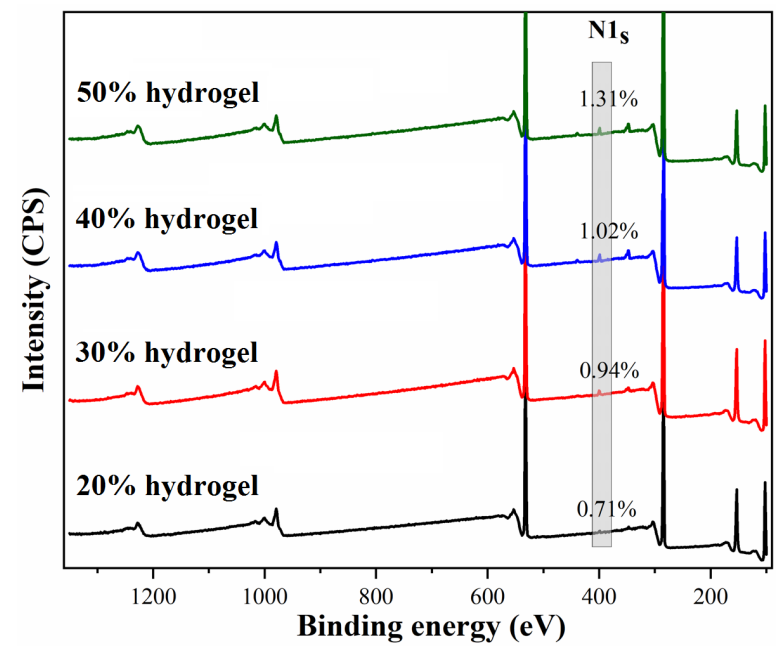

Figure 5. XPS survey spectra of the surfaces of silicone-hydrogel IPNs with hydrogel contents of $20 \%, 30 \%, 40 \%$ and $50 \%$ $(\mathrm{w} / \mathrm{w})$. N1s represents the increasing nitrogen content due to the increased number of spyropiron present at the surface

Water uptake and contact angle measurements: To evaluate if the surface wettability changes when the SP-photogated IPN is exposed to UV light, water uptake and water contact angle (CA) measurement was performed. Due to inhomogeneity of the cylindrical IPNs, it was not possible to conduct contact angle measurements on their surfaces. Therefore, a 
SPCOOH solution in THF was sputter-coated on cover slides and the wettability measured with contact angle meter (Dataphysics, Germany) before and after 1 min of UV irradiation. The measurements were performed at five different locations on each sample and the mean was taken as static CA. As shown in Figure 6, the SPCOOH isomerization after exposure to UV light, gave a CA decrease of $27^{\circ}$ (from $99.2^{\circ}$ to $72.2^{\circ}$ ), indicating increasing hydrophilicity of the samples.

As shown in Figure 7, the water uptake under UV light is higher for all SP grafted IPN compared to the samples at dark (UV off). This change stems from the hydrophobic layer provided by SP moieties at dark, which inhibit water from diffusing into the hydrogels of the IPNs. The isomerization of SP to MC acts as an opening of gate, which leads to diffusion of water into the interior hydrogel structure. These results are consistent with the CA measurements, which confirm that isomerisation of SPCOOH changes and leads to the hydrophilic MC conformation. It can be seen that the total water uptake is increasing with increasing hydrogel content, but the most interesting point is that as the hydrogel content of the SP-photogated IPN increases, the difference between water uptake with and without UV exposure decreases. For instance, the water uptake of the IPN with $20 \%$ hydrogel exposed to UV is almost twice as high as that of the same sample in the dark (UV off) (Figure 7a). On the other hand, the water uptake of the IPN with 50\% hydrogel increases only slightly upon UV irradiation (Figure 7d). This indicates that by increasing the hydrogel content, the SP molecules cannot inhibit the diffusion of water. This might be due to that the esterification of the HEMA alcohol groups was not complete or sufficient in the IPNs with high (40\% and 50\%) hydrogel content (Figure $7 \mathrm{c}$ and d).

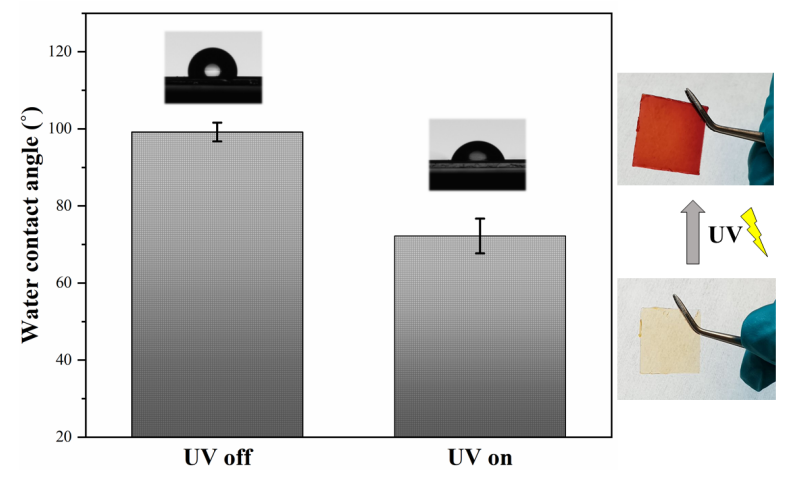

Figure 6. Contact angle measurement of SPCOOH-coated on a cover slide before and after UV exposure.

\subsection{Loading of SP-photogated IPNs with DOX}

The SP-photogated IPNs were loaded with DOX by passive diffusion. To select an appropriate loading solvent, it is important to consider that ${ }^{42}$ : i) The solvent should swell the hydrogel and not the silicone. ii) Polar solvents, like ethanol, drives the chemical equilibrium from SP towards MC, i.e. the energy level of MC is lower than that of SP, which causes the isomerization of SP to MC to be thermodynamically spontaneous and irreversible (negative photochromism) at dark $(\Delta \mathrm{G}<0)^{46-48}$. By choosing ethanol as the loading solvent, the hydrophobic SP moieties isomerize spontaneously to the hydrophilic MC form, which leads to hydrogel swelling and diffusion of DOX into the IPN. Thus, ethanol eliminates the need for UV irradiation to swell the samples during the loading step which otherwise might lead to photo-fatigue (loss of reversibility) of SP-photogated IPNs.
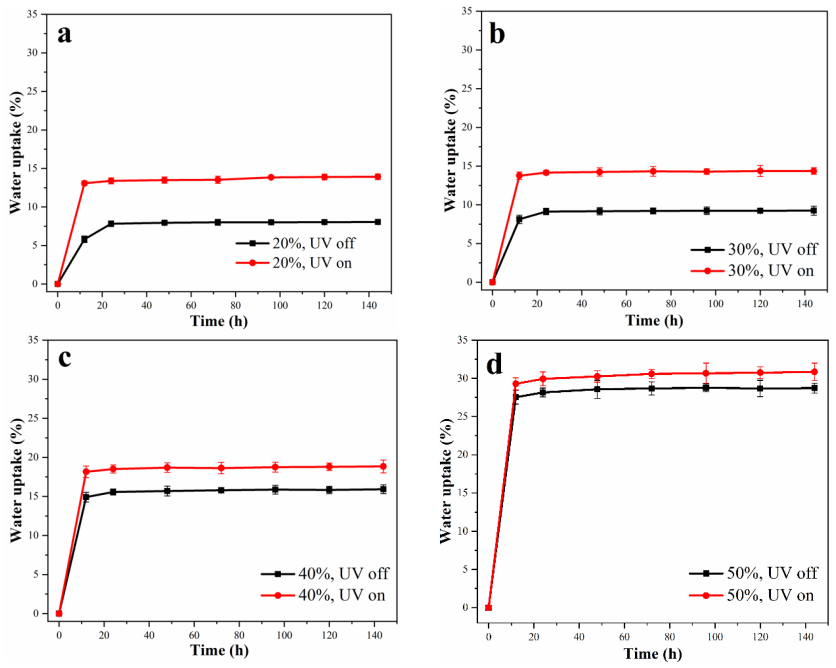

Figure 7. Water uptake measurements of SP-IPN grafts with: a) $20 \%$, b) $30 \%$, c) $40 \%$ and d) $50 \%$ (wt $\%$ ) hydrogel content with and without exposure to UV light. Data are presented for three identically treated samples as the mean \pm standard deviation $(n=3)$.

\subsection{Light-triggered release of DOX}

The premature release of DOX from the SP-grafted poly(HEMA-co-PEGMEA) IPN was compared with our previously developed SP-bulk IPN with poly(HEMA-coPEGMEA-co-SPMA) as guest polymer ${ }^{42}$ (both with $40 \%$ HEMA hydrogel content). Both IPNs were loaded with the same amount of DOX using the same method, as described in section 2.6. Figure 8 shows that the SP grafted IPN inhibits efficiently the premature (unwanted) release when UV is off; almost 15 times less DOX is released compared to the SP-bulk IPN.

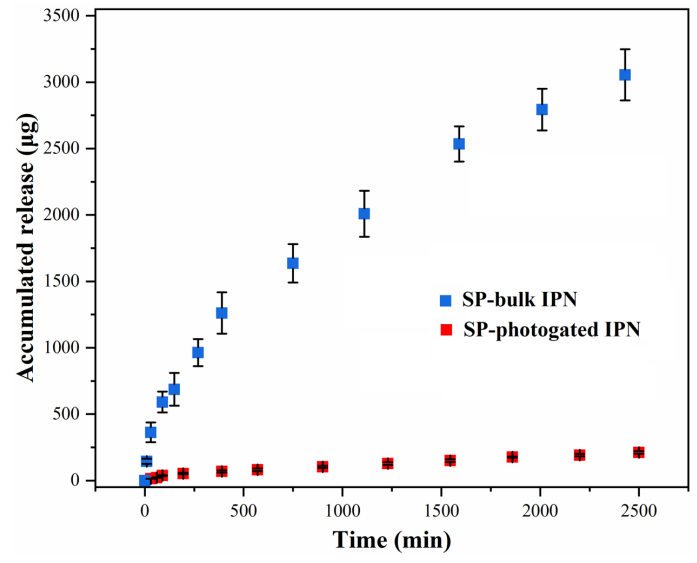

Figure 8. Comparing premature release of DOX from SP-bulk IPN and SP-photogated IPN from poly(HEMA-co-PEGMEAco-SPMA), each with $40 \%$ poly (HEMA-co-PEGMEA) hydrogel content. Data are presented for three identically treated samples as the mean \pm standard deviation $(n=3)$.

The triggered release of DOX from SP-grafted IPNs with different hydrogel content was investigated and the release 

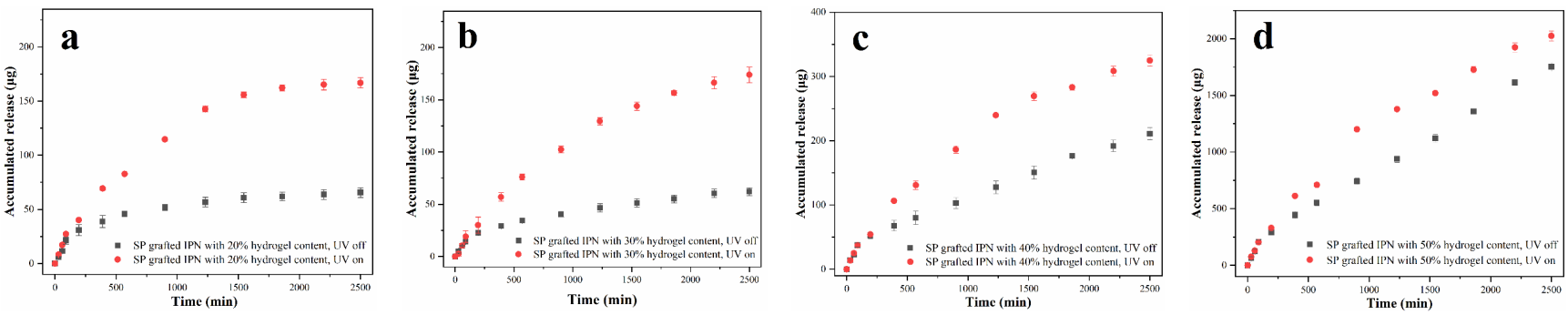

Figure 9. Light-triggered release profile of DOX from the SP-photogated IPNs with: a) $20 \%$, b) 30\%, c) $40 \%$ and d) $50 \%$ hydrogel content. Data are presented for three identically treated samples as the mean $\pm \operatorname{standard~deviation~}(n=3)$.

profiles shown in Figure 9, with and without exposure to UV light. It can be seen that for all IPN samples, the release is enhanced when exposed to UV. IPNs with the higher hydrogel content (40 and $50 \%$ HEMA) show however very high premature release (Figure 9c and d) whereas IPNs with the lower hydrogel content (20\% and 30\% HEMA) have almost three times higher triggered release than the premature release (Figure 9a and $\mathrm{b}$ ). This indicates that the hydrophobic SP molecules clearly have a gate-closing effect. The initial burst release with UV off might be due to residual DOX adsorbed on the surface of the IPNs, as the premature release was almost constant over time. These release results are consistent with the results of the water uptake measurements (Figure 7), which show that the water uptake of IPNs with high hydrogel content was high even without exposure to UV. It seems that, although the IPNs with high hydrogel content have more HEMA alcohol groups for SP attachment, the hydrophobic gate molecules on their surface cannot compete with the hydrophilicity and affinity of the hydrogel to water, which leads to swelling of the polymer and unwanted premature drug release. The release profiles in Figures 8 and 9ab indicate that the SP molecular gate is quite efficient, maybe too efficient, since the accumulated release for the best SP-grafted IPN with $20 \%$ hydrogel content (considering the ratio between accumulate and premature release) only reaches a maximum of 167 $\mu \mathrm{g}$ of DOX (Figure 9a). Overall, the release profiles in Figure 9 indicate that: i) a low hydrogel content leads to a low loading efficiency and subsequently a low accumulated drug release, however with an efficient SP molecular gate that inhibits premature release, and ii) a high hydrogel content provides a more hydrophilic environment and therefore more swelling of the IPN and higher accumulated release, but with a less efficient SP molecular gate, leading to increased premature release. Our previous work indicates that the premature and accumulated release can potentially be tuned by playing around with guest polymer composition, varying the hydrophilicity/hydrophobicity of the IPN in respect to the requirements and the drugs to be released ${ }^{42}$.

\section{CONCLUSION}

Photo-responsive DDS based on silicone-hydrogel IPNs were successfully achieved using the combination of $\mathrm{scCO}_{2}$ technology and surface modification by grafting of photo-responsive SPCOOH on the IPN surface to generate photo-switchable molecular gate based IPNs. This novel system could address the problems associated with the light-triggered release systems that has been designed so far. Where, the triggered release is not based on the disruption and degradation of the drug carrier, but it was achieved by exploiting the change in the wetting behaviour of surface by the SP hydrophobic-tohydrophilic switch. Moreover, the hydrogel which is impregnated into the silicone elastomer provides the opportunity to deliver hydrophilic drugs on-demand. Comparing post-modification of silicon-hydrogel IPNs with SP moieties grafted on the surface (SP-photogated IPN) and SP co-polymerised inside the bulk of the silicon-hydrogel IPN (SP-bulk IPN) demonstrates that the SP-photogated IPN had much less premature DOX release compared to the SP-bulk IPN with the same overall hydrogel and DOX content. The SP molecules of the SP-photogated IPN provide a hydrophobic layer around the silicon hydrogel IPN, which can inhibit the diffusion of water and release of drug from the material. Upon irradiation of UV light, the "closed" SP gate molecules convert to the "open" hydrophilic MC state, which causes surface wetting and increased diffusion of water into the IPN; therefore, leading to the release of DOX. The SP gate-closing efficiency very much depends on the hydrogel content. IPNs with high hydrogel content $(40 \%$ and $50 \%)$, which in spite of having a higher ratio of SP molecules on the surface, show a substantially higher premature release with limited light-triggered drug release. IPNs with the lower hydrogel content (20\% and 30\%) showed efficient SP-photogated DOX release with a substantial difference between the light triggered accumulated release and premature release, however, with lower accumulated release compared to IPNs with the higher hydrogel content. From our previous research, we know that the premature and accumulated release could in principle be adjusted simply by tuning the polymer composition in the IPN, depending on the requirements and the lipophilicity of the drug to be released. This molecular gated strategy may be promising for future development of on-demand drug or compound delivery for various applications.

\section{AUTHOR INFORMATION}

\section{Corresponding Author}

*corresponding authors: jemn@dtu.dk; ma@biomodics.com

\section{Author Contributions}

The manuscript was written through contributions of all authors. All authors have given approval to the final version of the manuscript.

\section{ACKNOWLEDGMENT}

This project was funded by the European Union Horizon 2020 Programme (H2020-MSCA-ITN-2016) under the Marie Skłodowska-Curie Innovative Training Network, project Training4CRM with Grant Agreement No.722779.

\section{REFERENCES}

(1) Mura, S.; Nicolas, J.; Couvreur, P. Stimuli-Responsive Nanocarriers for Drug Delivery. Nat. Mater. 2013, 12 (11), 
991-1003. https://doi.org/10.1038/nmat3776.

Hrubý, M.; Filippov, S. K.; Štěpánek, P. Smart Polymers in Drug Delivery Systems on Crossroads: Which Way Deserves Following? European Polymer Journal. 2015, pp 82-97. https://doi.org/10.1016/j.eurpolymj.2015.01.016.

Langer, R. New Methods of Drug Delivery. Science (80-. ). 1990, 249 (4976), 1527-1533. https://doi.org/10.1126/science.2218494.

(4) Liu, D.; Yang, F.; Xiong, F.; Gu, N. The Smart Drug Delivery System and Its Clinical Potential. Theranostics. Ivyspring International Publisher 2016, pp 1306-1323. https://doi.org/10.7150/thno.14858.

(5) Alvarez-Lorenzo, C.; Concheiro, A. Smart Drug Delivery Systems: From Fundamentals to the Clinic. Chem. Commun. 2014, $50 \quad$ (58), $\quad$ 7743-7765. https://doi.org/10.1039/C4CC01429D.

(6) Hossen, S.; Hossain, M. K.; Basher, M. K.; Mia, M. N. H.; Rahman, M. T.; Uddin, M. J. Smart Nanocarrier-Based Drug Delivery Systems for Cancer Therapy and Toxicity Studies: A Review. J. Adv. Res. 2019, 15, 1-18. https://doi.org/10.1016/j.jare.2018.06.005.

(7) Chen, J. K.; Chang, C. J. Fabrications and Applications of Stimulus-Responsive Polymer Films and Patterns on Surfaces: A Review. Materials. 2014, pp 805-875. https://doi.org/10.3390/ma7020805.

(8) Linsley, C. S.; Wu, B. M. Recent Advances in LightResponsive on-Demand Drug-Delivery Systems. Therapeutic Delivery. Future Medicine Ltd. February 1, 2017, pp 89-107. https://doi.org/10.4155/tde-2016-0060.

(9) Sahu, A.; Kim, M.; Ryu, J.; Son, J. G.; Lee, E.; Noh, D. Y.; Tae, G. Nanographene Oxide as a Switch for CW/Pulsed NIR Laser Triggered Drug Release from Liposomes. Mater. Sci. Eng. C 2018, 82, 19-24. https://doi.org/10.1016/j.msec.2017.08.057.

(10) Zhou, Y.; Chen, R.; Yang, H.; Bao, C.; Fan, J.; Wang, C.; Lin, Q.; Zhu, L. Light-Responsive Polymersomes with a ChargeSwitch for Targeted Drug Delivery. J. Mater. Chem. B 2020, 8 (4), 727-735. https://doi.org/10.1039/c9tb02411e.

(11) Priya James, H.; John, R.; Alex, A.; Anoop, K. R. Smart Polymers for the Controlled Delivery of Drugs - a Concise Overview. Acta Pharm. Sin. B 2014, 4 (2), 120-127. https://doi.org/10.1016/j.apsb.2014.02.005.

(12) Schumers, J. M.; Fustin, C. A.; Gohy, J. F. Light-Responsive Block Copolymers. Macromol. Rapid Commun. 2010, 31 (18), 1588-1607. https://doi.org/10.1002/marc.201000108.

(13) Kelley, E. G.; Albert, J. N. L.; Sullivan, M. O.; Epps, III, T. H. Stimuli-Responsive Copolymer Solution and Surface Assemblies for Biomedical Applications. Chem. Soc. Rev. 2013, 42 (17), 7057. https://doi.org/10.1039/c3cs35512h.

(14) Jia, S.; Fong, W.-K.; Graham, B.; Boyd, B. J. Photoswitchable Molecules in Long-Wavelength Light-Responsive Drug Delivery: From Molecular Design to Applications. Chem. Mater. $\quad 2018, \quad 30 \quad$ (9), $2873-2887$. https://doi.org/10.1021/acs.chemmater.8b00357.

(15) Minkin, V. I. Photo-, Thermo-, Solvato-, and Electrochromic Spiroheterocyclic Compounds. Chem. Rev. 2004, 104 (5), 2751-2776. https://doi.org/10.1021/cr020088u.

(16) Berkovic, G.; Krongauz, V.; Weiss, V. Spiropyrans and Spirooxazines for Memories and Switches. Chem. Rev. 2000, 100 (5), 1741-1753. https://doi.org/10.1021/cr9800715.

(17) Cardano, F.; Del Canto, E.; Giordani, S. Spiropyrans for LightControlled Drug Delivery. Dalt. Trans. 2019, 48 (41), 1553715544. https://doi.org/10.1039/c9dt02092f.

(18) Klajn, R. Spiropyran-Based Dynamic Materials. Chem. Soc. Rev. 2014, $43 \quad$ (1), 148-184. https://doi.org/10.1039/C3CS60181A.

(19) Ma, T.; Walko, M.; Lepoitevin, M.; Janot, J. M.; Balanzat, E.; Kocer, A.; Balme, S. Combining Light-Gated and PHResponsive Nanopore Based on PEG-Spiropyran Functionalization. Adv. Mater. Interfaces 2018, 5 (2), 1701051. https://doi.org/10.1002/admi.201701051.

(20) Ali, A. A.; Kharbash, R.; Kim, Y. Chemo- and Biosensing Applications of Spiropyran and Its Derivatives - A Review. Anal. Chim. Acta 2020. https://doi.org/10.1016/j.aca.2020.01.057.

(21) Son, S.; Shin, E.; Kim, B.-S. Light-Responsive Micelles of
Spiropyran Initiated Hyperbranched Polyglycerol for Smart Drug Delivery. Biomacromolecules 2014, 15 (2), 628-634. https://doi.org/10.1021/bm401670t.

(22) Lee, H. Il; Wu, W.; Oh, J. K.; Mueller, L.; Sherwood, G.; Peteanu, L.; Kowalewski, T.; Matyjaszewski, K. Light-Induced Reversible Formation of Polymeric Micelles. Angew. Chemie Int. $\quad E d$. 2007, $46 \quad$ (14), 2453-2457. https://doi.org/10.1002/anie.200604278.

(23) Yuan, W.; Gao, X.; Pei, E.; Li, Z. Light- and PH-Dually Responsive Dendrimer-Star Copolymer Containing Spiropyran Groups: Synthesis, Self-Assembly and Controlled Drug Release. Polym. Chem. 2018, 9 (26), 3651-3661. https://doi.org/10.1039/C8PY00721G.

(24) Yu, L. X.; Liu, Y.; Chen, S. C.; Guan, Y.; Wang, Y. Z. Reversible Photoswitching Aggregation and Dissolution of Spiropyran-Functionalized Copolymer and Light-Responsive FRET Process. Chinese Chem. Lett. 2014, 25 (3), 389-396. https://doi.org/10.1016/j.cclet.2013.12.014.

(25) Yang, Z.; Zou, H.; Liu, H.; Xu, W.; Zhang, L. Self-Assembly and Drug Release Control of Dual-Responsive Copolymers Based on Oligo(Ethylene Glycol)Methyl Ether Methacrylate and Spiropyran. Iran. Polym. J. 2019, 28 (1), 39-49. https://doi.org/10.1007/s13726-018-0677-7.

(26) Zou, H.; Liu, H. Synthesis of Thermal and Photo DualResponsive Amphiphilic Random Copolymer via Atom Transfer Radical Polymerization and Its Control Release of Doxorubicin. Int. J. Polym. Mater. Polym. Biomater. 2017, 66 (18),

https://doi.org/10.1080/00914037.2017.1291515.

(27) Chen, C.-J.; Jin, Q.; Liu, G.-Y.; Li, D.-D.; Wang, J.-L.; Ji, J. Reversibly Light-Responsive Micelles Constructed via a Simple Modification of Hyperbranched Polymers with Chromophores. Polymer (Guildf). 2012, 53 (17), 3695-3703. https://doi.org/10.1016/j.polymer.2012.06.024.

(28) Jin, Q.; Liu, G.; Ji, J. Micelles and Reverse Micelles with a Photo and Thermo Double-Responsive Block Copolymer. $J$. Polym. Sci. Part A Polym. Chem. 2010, 48 (13), 2855-2861. https://doi.org/10.1002/pola.24062.

(29) Chen, S.; Liu, H.; Hu, J.; Zou, H.; He, Y. Self-Assembly and Morphology Transition of Amphipathic Spiropyran-Based Random Copolymers to Control Drug Release. Des. Monomers Polym. 2016, 19 (8), $\quad 730-739$. https://doi.org/10.1080/15685551.2016.1209632.

(30) Pugachev, A. D.; Ozhogin, I. V.; Lukyanova, M. B.; Lukyanov, B. S.; Rostovtseva, I. A.; Dorogan, I. V.; Makarova, N. I.; Tkachev, V. V.; Metelitsa, A. V.; Aldoshin, S. M. Visible to Near-IR Molecular Switches Based on Photochromic Indoline Spiropyrans with a Conjugated Cationic Fragment. Spectrochim. Acta - Part A Mol. Biomol. Spectrosc. 2020, 230, 118041. https://doi.org/10.1016/j.saa.2020.118041.

(31) Liu, C.; Zhang, Y.; Liu, M.; Chen, Z.; Lin, Y.; Li, W.; Cao, F.; Liu, Z.; Ren, J.; Qu, X. A NIR-Controlled Cage Mimicking System for Hydrophobic Drug Mediated Cancer Therapy. Biomaterials 2017, 139, 151-162. https://doi.org/10.1016/j.biomaterials.2017.06.008.

(32) Chen, L.; Wang, W.; Su, B.; Wen, Y.; Li, C.; Zhou, Y.; Li, M.; Shi, X.; Du, H.; Song, Y.; Jiang, L. A Light-Responsive Release Platform by Controlling the Wetting Behavior of Hydrophobic Surface. ACS Nano 2014, 8 (1), 744-751. https://doi.org/10.1021/nn405398d

(33) Xing, Q.; Li, N.; Chen, D.; Sha, W.; Jiao, Y.; Qi, X.; Xu, Q.; $\mathrm{Lu}$, J. Light-Responsive Amphiphilic Copolymer Coated Nanoparticles as Nanocarriers and Real-Time Monitors for Controlled Drug Release. J. Mater. Chem. B 2014, 2 (9), 1182 1189. https://doi.org/10.1039/c3tb21269f.

(34) Aznar, E.; Casasús, R.; García-Acosta, B.; Dolores Marcos, M.; Martínez-Máñez, R.; Sancenón, F.; Soto, J.; Amorós, P. Photochemical and Chemical Two-Channel Control of Functional Nanogated Hybrid Architectures. Adv. Mater. 2007, 19 (17), 2228-2231. https://doi.org/10.1002/adma.200601958. Klein, K.; Grønnemose, R. B.; Alm, M.; Brinch, K. S.; Kolmos, H. J.; Andersen, T. E. Controlled Release of Plectasin NZ2114 from a Hybrid Silicone-Hydrogel Material for Inhibition of Staphylococcus Aureus Biofilm. Antimicrob. Agents Chemother. 2017, 61 (7). https://doi.org/10.1128/AAC.00604- 
17.

Stenger, M.; Klein, K.; Grønnemose, R. B.; Klitgaard, J. K.; Kolmos, H. J.; Lindholt, J. S.; Alm, M.; Thomsen, P.; Andersen, T. E. Co-Release of Dicloxacillin and Thioridazine from Catheter Material Containing an Interpenetrating Polymer Network for Inhibiting Device-Associated Staphylococcus Aureus Infection. J. Control. Release 2016, 241, 125-134. https://doi.org/10.1016/j.jconrel.2016.09.018.

(37) Riber, L.; Burmølle, M.; Alm, M.; Milani, S. M.; Thomsen, P.; Hansen, L. H.; Sørensen, S. J. Enhanced Plasmid Loss in Bacterial Populations Exposed to the Antimicrobial Compound Irgasan Delivered from Interpenetrating Polymer Network Silicone Hydrogels. Plasmid 2016, 87-88, 72-78. https://doi.org/10.1016/j.plasmid.2016.10.001.

(38) Ramstedt, M.; Ribeiro, I. A. C.; Bujdakova, H.; Mergulhão, F. J. M.; Jordao, L.; Thomsen, P.; Alm, M.; Burmølle, M.; Vladkova, T.; Can, F.; Reches, M.; Riool, M.; Barros, A.; Reis, R. L.; Meaurio, E.; Kikhney, J.; Moter, A.; Zaat, S. A. J.; Sjollema, J. Evaluating Efficacy of Antimicrobial and Antifouling Materials for Urinary Tract Medical Devices: Challenges and Recommendations. Macromolecular Bioscience. May 18, 2019, p 1800384. https://doi.org/10.1002/mabi.201800384.

(39) Mohanty, S.; Alm, M.; Hemmingsen, M.; Dolatshahi-Pirouz, A.; Trifol, J.; Thomsen, P.; Dufva, M.; Wolff, A.; Emnéus, J. 3D Printed Silicone-Hydrogel Scaffold with Enhanced Physicochemical Properties. Biomacromolecules 2016, 17 (4), 1321-1329. https://doi.org/10.1021/acs.biomac.5b01722.

Steffensen, S. L.; Vestergaard, M. H.; Møller, E. H.; Groenning, M.; Alm, M.; Franzyk, H.; Nielsen, H. M. Soft Hydrogels Interpenetrating Silicone - A Polymer Network for Drug-Releasing Medical Devices. J. Biomed. Mater. Res. - Part B Appl. Biomater. 2016, 104 (2), 402-410. https://doi.org/10.1002/jbm.b.33371.

(41) Steffensen, S. L.; Vestergaard, M. H.; Groenning, M.; Alm, M.; Franzyk, H.; Nielsen, H. M. Sustained Prevention of Biofilm Formation on a Novel Silicone Matrix Suitable for Medical Devices. Eur. J. Pharm. Biopharm. 2015, 94, 305-311. https://doi.org/10.1016/j.ejpb.2015.05.014.

(42) Ghani, M.; Heiskanen, A.; Kajtez, J.; Thomsen, P.; Kristensen, A.; Žukauskas, A.; Alm, M.; Emnéus, J. On-Demand
Reversible Light-Triggered Interpenetrating Polymer Network Based Drug Delivery System Using the SpiropyranMerocyanine Hydrophobicity Switch. Submitt. to Biofabrication 2020.

(43) Chen, J.; Zeng, F.; Wu, S.; Zhao, J.; Chen, Q.; Tong, Z. Reversible Fluorescence Modulation through Energy Transfer with ABC Triblock Copolymer Micelles as Scaffolds. Chem. Commun. 2008, No. 43, 5580-5582. https://doi.org/10.1039/b810677k.

(44) Baumann, L.; Schöller, K.; De Courten, D.; Marti, D.; Frenz, M.; Wolf, M.; Rossi, R. M.; Scherer, L. J. Development of Light-Responsive Porous Polycarbonate Membranes for Controlled Caffeine Delivery. RSC Adv. 2013, 3 (45), 23317 23326. https://doi.org/10.1039/c3ra44399j.

(45) Nordin, R.; Lazim, A. M.; Hasbullah, S. A. Spectral and Photochromic Study of Spiropyran. In AIP Conference Proceedings; 2013; Vol. 1571, pp 817-821. https://doi.org/10.1063/1.4858756.

(46) Abdollahi, A.; Sahandi-Zangabad, K.; Roghani-Mamaqani, H. Light-Induced Aggregation and Disaggregation of StimuliResponsive Latex Particles Depending on Spiropyran Concentration: Kinetics of Photochromism and Investigation of Reversible Photopatterning. Langmuir 2018, 34 (46), 13910 13923. https://doi.org/10.1021/acs.langmuir.8b02296.

Abdollahi, A.; Alinejad, Z.; Mahdavian, A. R. Facile and Fast Photosensing of Polarity by Stimuli-Responsive Materials Based on Spiropyran for Reusable Sensors: A PhysicoChemical Study on the Interactions. J. Mater. Chem. C 2017, 6588 (5), 6588-6600. https://doi.org/10.1039/c7tc02232h.

(48) Tian, W.; Tian, J. An Insight into the Solvent Effect on Photo-, Solvato-Chromism of Spiropyran through the Perspective of Intermolecular Interactions. Dye. Pigment. 2014, 105, 66-74. https://doi.org/10.1016/j.dyepig.2014.01.020.

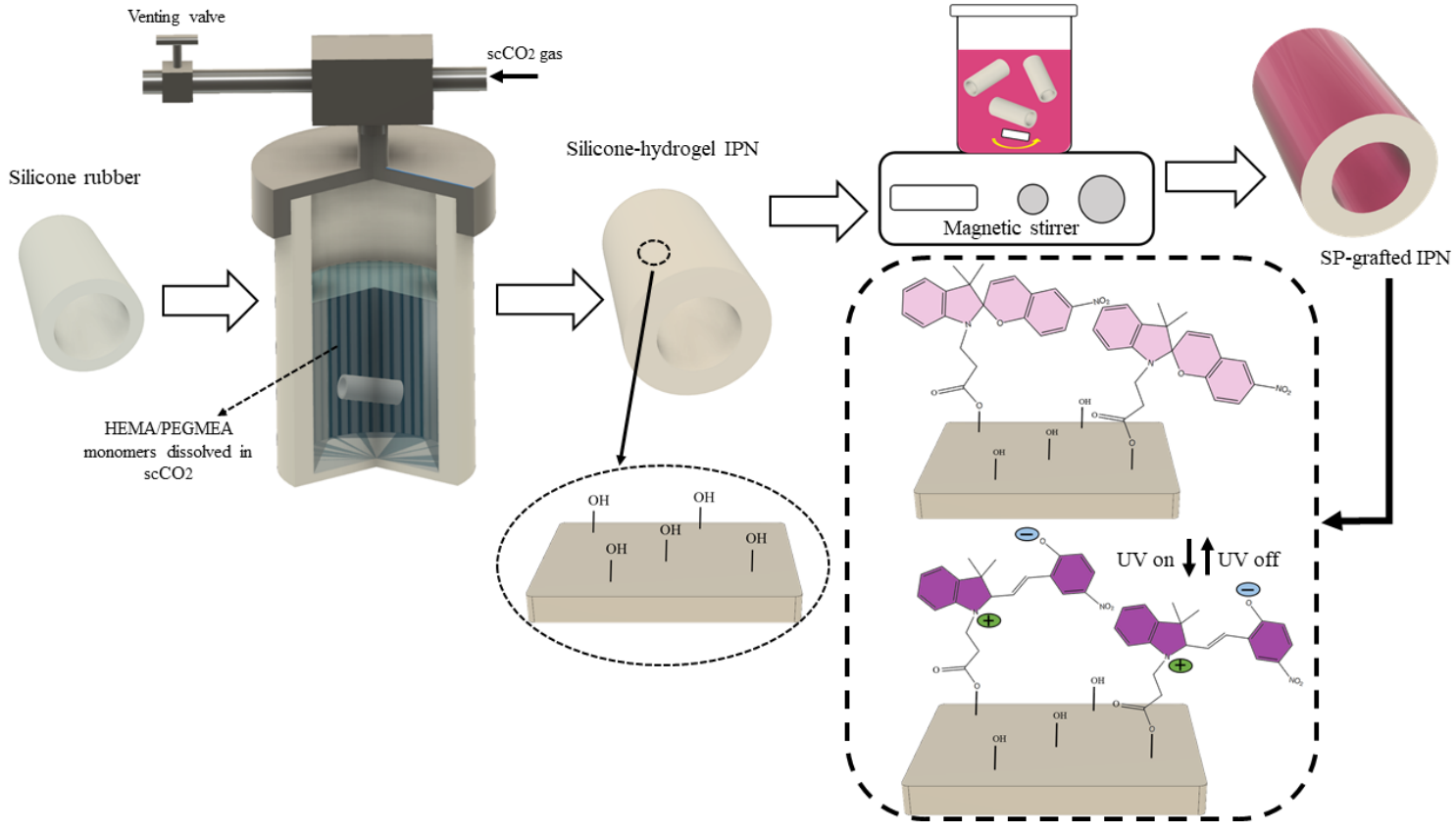

